\title{
Transparency of Positive Surgical Margins after Radical Prostatectomy in the Benelux
}

\author{
CKM Vermeulen* and LMCL Fossion
}

Department of Urology, Máxima Medical Centre, De Run 4600, 5504 DB Veldhoven, Netherlands

Received: January 22, 2016; Accepted: March 01, 2016; Published: March 09, 2016

*Corresponding author: CKM Vermeulen, Bachelor of Science, Student Master of Medicine, Faculty of Health, Medicine and Life Sciences, Maastricht University Universiteitssingel 60, 6229 ER Maastricht, Netherlands, T: +316 18114029; E-mail: ckm.vermeulen@student.maastrichtuniversity.nl

\begin{abstract}
Objective: The oncologic outcome after radical prostatectomy is negatively influenced by Positive Surgical Margins (PSM), as it increases the risk of disease recurrence. Analysis of the incidence of PSM could contribute in improving the surgical treatment of prostate cancer. To stimulate more transparency in this topic, we studied our own population.
\end{abstract}

Materials and Methods: 355 EERPE (Endoscopic Extraperitoneal Radical Prostatectomy) patients from a singlesurgeon database were gathered in the period of 2006-2014 and analyzed retrospectively. Outcome was measured postoperatively in pathologically confirmed PSM. A chi-square test was performed to objectify the relation between PSM and tumor stage and nerve sparing procedures. A literature study was performed to evaluate numbers of other urologic departments in Belgium and the Netherlands.

Results: $36.6 \%$ of all EERPEs resulted in PSM, $23.3 \%$ of the pT2 tumors and $63.0 \%$ of the pT3 tumors. Advanced tumor stage was found to be a significant risk factor for PSM. Our results are consistent with the few results available in literature.

Conclusion: Our numbers prove the relation between PSM and tumor stage. At the moment, existing data in the Belgium and the Netherlands are insufficient for critical evaluation of the oncologic outcome of radical prostatectomy. In order to assess and improve the surgical treatment of prostate cancer, more transparency regarding PSM is necessary.

Keywords: Radical prostatectomy; Oncologic outcome; Positive surgical margin; Prostate cancer; Netherlands; Belgium

\section{Abbreviations \\ cT: Clinical Tumor Stage; EERPE: Endoscopic Extraperitoneal Radical Prostatectomy; HiFu: High-Intensity Focused Ultrasound; IKNL: Integral Cancer Centre of the Netherlands; LPLND: Laparoscopic Pelvic Lymph Node Dissection; LRP: Laparoscopic Radical Prostatectomy; MMC: Máxima Medical Centre; MRI: Magnetic Resonance Imaging; NS: Nerve Sparing ; NVU: Dutch Association of Urology; OR: Operating Room; PSA: Prostate Specific Antigen; PSM: Positive Surgical Margin; pT: Pathological Tumor Stage; RALP: Robot Assisted Laparoscopic Prostatectomy; RP: Radical Prostatectomy; RRP: Retropubic}

Radical Prostatectomy; rT: Radiological Tumor Stage; TNM: TNM Classification of Malignant Tumors; Metric units were used for the measurements of length, height, weight and volume.

\section{Introduction}

Positive Surgical Margins (PSM) in Radical Prostatectomy (RP) are considered an adverse pathologic feature [1-4]; however, their association with clinical progression and biochemical recurrence remains controversial [5,6]. Attention to surgical detail and precise preoperative tumor staging is essential to minimize the risk of PSM and thus minimize the potential risk of disease recurrence [6-8]. The likelihood of PSM is strongly influenced by the surgeon's experience irrespective of the surgical approach. Numbers of PSM range from 16-63\% in the available literature in the Benelux, and PSM-rate is higher in men with a more advanced pathologic tumor stage. These numbers show that there is still room for improvement [9-15].

Beside numeric requirements (e.g. high volume surgeons), transparency of surgical margins can aid to interpret and improve the quality of care. Critical evaluation of the pathological outcome could provide new insights in the surgical treatment of prostate cancer and publication of the PSM-rate per surgeon and per center can be a first step. Permanent quality analysis, certainly during the learning curve, might help to improve surgical strategy and skills for each surgeon [16]. The aim of this study is to stimulate more transparency regarding this subject, starting with presenting our own series.

\section{Patients and Methods}

In the period from 2006 until 2014, 385 Endoscopic Extraperitoneal Radical Prostatectomy (EERPE) were performed in our department (2006-2008 in Bernhoven hospital and 2008-2014 in Máxima Medical Center). All patients, eligible for radical prostatectomy, were included and underwent an EERPE as described by Stolzenburg's [17], combined with lymph node dissection in all intermediate and high risk patients according to the d'Amico classification. Eligibility criteria were: male patients with life expectancy of at least 10 years, with local or locally advanced prostate cancer and eligible for curative treatment, 


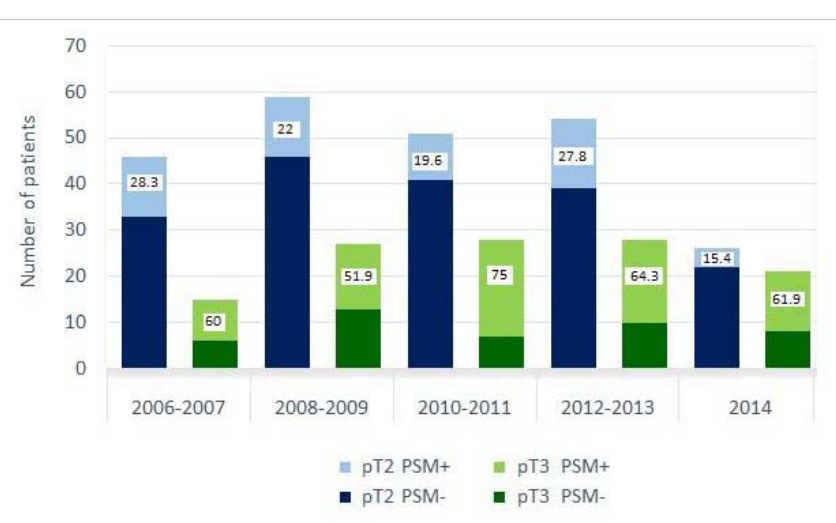

Figure 1: Evolution of the surgical margins in EERPE in respective to the learning curve of a single surgeon (LF) over the period 2006-2014. $\%$ of the PSM rates over the time are illustrated in the columns

without contra-indication for surgery. In this period, eighteen EERPE procedures were performed by a second urologist. These patients were excluded from this study to avoid bias of intersurgical experience, creating a single surgeon database. For each patient, the pathological examination of the tumor was reviewed. Patients with a pathological T2 or T3 stage prostate adenocarcinoma were included in our series. All other pathological tumor stages were excluded $(n=12)$.

Patient characteristics such as age, Gleason score, Prostate Specific Antigen (PSA), OR-time, tumor stage, risk classification, estimated blood loss, the surgical technique (nerve sparing or not), number treated per year, pathology reports, and peri-and postoperative data were gathered prospectively since 2006. Thepatients were analyzed retrospectively. Outcome was measured in the presence or absence of PSM, which was stated in the pathology report. For this study, patients were followed from the day of surgery to hospital discharge. No analyses regarding survival or prognostic value of PSM were included in this study.

We converted the patient data to an anonymous SPSS database for sufficient analysis. Descriptive statistics were used in order to interpret the patient characteristics pre-, peri and postoperatively in the total population, the patient group with PSM and the patient group without PSM. Differences between the PSM group and the non-PSM group were calculated using the Chisquare test for the categorical variables, the independent $\mathrm{T}$ test for continuous variables and the Mann-Whitney test was used to calculate differences in Gleason score. We calculated risk factors for PSM with an odds ratio using logistic regression.

To compare our results to other series in the Benelux, a PubMed and Cochrane search was performed using the items: radical prostatectomy, LRP (Laparoscopic RP), EERPE, RALP (Robot Assisted Laparoscopic Prostatectomy), oncologic outcome, positive surgical margin, PSM, urology, prostate cancer, the Netherlands, and Belgium.

\section{Results}

From 2006-2014, we totally included 355 patients which underwent an EERPE. Pre-, peri, and postoperative patient characteristics are illustrated in table 1 . The median age of the patients was 65 years at the time of operation. Pre-operative Gleason scores according to the TNM classification of 2002 ranged from 4-10 with a median of 6 . The mean pre-operative PSA was $11.9 \mathrm{ug} / \mathrm{L}$ and varied between 0.87 and $77 \mathrm{ug} / \mathrm{L}$ with a median of 8.75. Patients with PSM had a significantly higher median PSA at baseline ( $p=0.012)$ According to the d'Amico classification, 93 patients met the low risk criteria (26.2\%), 113 patients had an intermediate risk (31.8\%) and 149 patients were consistent with the high risk criteria (42.0\%). The d'Amico risk was significantly different in patients with and without PSM ( $p=$ 0.003). Pre-operative clinical staging by urologist and radiologist resulted in 151 cT1c tumors (42.5\%), 80 patients with a cT2a$2 \mathrm{~b}$ tumor $(22.5 \%)$ and 124 patients with a cT2c-3 tumor (34.9\%). 12 patients were diagnosed with an rT3-stage tumor; in these patients the MRI showed extra capsular disease preoperative (3.4\%). Of these 12 patients, $58.3 \%$ were confirmed as a pathological T3 stadium $(\mathrm{n}=7)$. In the other 5 patients, extra capsular extension was not pathologically confirmed.

Perioperative, 82 patients underwent bilateral nerve sparing NS technique and in 69 patients unilateral NS was performed. Laparoscopic Pelvic Lymph Node Dissection (LPLND) was performed in 226 patients (63.7\%). The median estimated blood loss was $400 \mathrm{cc}$ and ranged from $10-4000 \mathrm{cc}$ with an outlier of $6600 \mathrm{cc}$. This patient encountered a rectal injury and severe bleeding perioperative. The OR took on average 210 minutes, ranging from 103 - 430 minutes with a median of 200 minutes, depending on performance of LPLND (median160 min for EERPE only and $220 \mathrm{~min}$ for EERPE + LPLND). When performing lymph node dissection, the OR time was increased significantly $(p<0.001)$. After 4.6 days on average, with a median of 3 days, patients were fit to leave the hospital. Hospital stay ranged from 1-30 days with an outlier of 56 days. This patient suffered from severe comorbidities. His recovery was complicated by a postoperatively diagnosed rectal injury with sepsis, which was treated with relaparotomy twice and colostoma.

Pathology report showed 236 patients with a pT2-tumor and 119 patients with a pT3-tumor. The PSM-rate for the whole cohort was $36.6 \%$. Patients who underwent bilateral NS surgery had $34.6 \%$ chance of PSM and those with unilateral NS surgery had $30.4 \%$ of PSM. Patients with pT2-stage tumor had $23.3 \%$ PSM and patients with pT3-stage tumor $63.0 \%$ PSM. The results are displayed in table 2.

Table 2 gives insight into the PSM rates of the patients treated in the Bernhoven and MMC hospital from 2006 until 2014. The total PSM scores have been improving moderately over the years, which indicate a reasonable learning curve of the surgeon. However, comparing the PSM-rates of the two stages (pT2 and pT3) separately, an evident learning curve could be identified. The PSM rates in the pT2-tumors have improved from $33.3 \%$ in 2006 to $15.4 \%$ in 2014. Also the PSM-rates in the pT3-tumor have decreased from $71.4 \%$ in 2006 to $63.0 \%$ in 2014 . Tumor stage is a proven risk factor for PSM and the ratio pT3:pT2 has increased over the years, which affects the total scores negatively. Therefore, the total scores only are not suitable to judge the presence of a learning curve in our series, which is clearly there. 


\begin{tabular}{|c|c|c|c|c|}
\hline Patient Characteristics & Total $(n=355)$ & PSM+ $(n=130)$ & PSM- $(n=225)$ & $p$-value \\
\hline Pre-operative & Median (range) & Median (range) & Median (range) & \\
\hline Age (yrs) & $65(44-76)$ & $66(49-76)$ & $64(44-75)$ & 0.063 \\
\hline Gleason score & $6(4-10)$ & $6(4-10)$ & $6(4-10)$ & 0.363 \\
\hline PSA (ug/L) & 8.75 (0.87-77) & $9.5(2-77)$ & $8.3(0.9-71)$ & 0.012 \\
\hline d'Amico risk & N (\%) & N (\%) & N (\%) & 0.003 \\
\hline Low & $93(26.2)$ & $26(20)$ & $67(29.8)$ & \\
\hline Intermediate & $113(31.8)$ & $34(26.2)$ & $79(35.1)$ & \\
\hline High & $149(42.0)$ & $70(53.8)$ & $79(35.1)$ & \\
\hline Perioperative & Median (range) & Median (range) & Median (range) & \\
\hline Estimated blood loss (cc) & $400(10-6600)$ & $400(10-4000)$ & $350(20-6600)$ & 0.586 \\
\hline Overall OR time (min) & $200(103-430)$ & $212(105-430)$ & $197(103-400)$ & 0.279 \\
\hline OR time (min) No LPLND & $160(103-364)$ & $164(105-364)$ & $159(103-318)$ & \\
\hline OR time (min) LPLND & $220(130-430)$ & $220(142-430)$ & $220(130-400)$ & \\
\hline Nerve sparing procedure & N (\%) & N (\%) & N (\%) & 0.334 \\
\hline Unilateral & $69(19.4)$ & $21(16.2)$ & $48(21.3)$ & \\
\hline Bilateral & $82(23.1)$ & $28(21.5)$ & $54(24.0)$ & \\
\hline Total & $151(42.5)$ & 49 (37.7) & $102(45.3)$ & \\
\hline LPLND & $226(63.7)$ & $88(67.7)$ & $138(61.3)$ & 0.231 \\
\hline Postoperative p-stage & & & & $<0.0001$ \\
\hline pT2 & $236(66.5)$ & $55(42.3)$ & $181(80.4)$ & \\
\hline pT3 & $119(33.5)$ & 75 (57.7) & $44(19.6)$ & \\
\hline Total & $355(100)$ & $130(36.6)$ & $225(63.4)$ & \\
\hline
\end{tabular}

Table 2: Data of positive surgical margins (PSM) in EERPEs, performed by single surgeon (LF) over the period 2006 - 2014, MMC data.

\begin{tabular}{|c|c|c|c|c|c|c|c|c|c|c|}
\hline \multirow[t]{2}{*}{ Year } & \multicolumn{2}{|l|}{ Total } & \multicolumn{2}{|c|}{ NS unilateral } & \multicolumn{2}{|c|}{ NS bilateral } & \multicolumn{2}{|l|}{ pT2 } & \multicolumn{2}{|l|}{ pT3 } \\
\hline & $\mathrm{N}$ & PSM \% & $\mathrm{N}$ & PSM \% & $\mathrm{N}$ & PSM \% & $\mathrm{N}$ & PSM \% & $\mathrm{N}$ & PSM \% \\
\hline $2006^{*}$ & 23 & 43.5 & 7 & 42.9 & 3 & 33.3 & 16 & 31.3 & 7 & 71.4 \\
\hline $2007^{*}$ & 38 & 31.6 & 14 & 21.4 & 7 & 28.6 & 30 & 26.7 & 8 & 50.0 \\
\hline 2008 & 41 & 41.5 & 8 & 62.5 & 8 & 37.5 & 26 & 26.9 & 15 & 66.7 \\
\hline 2009 & 45 & 22.2 & 9 & 22.2 & 7 & 28.6 & 33 & 18.2 & 12 & 33.3 \\
\hline 2010 & 40 & 37.5 & 9 & 22.2 & 9 & 22.2 & 26 & 19.2 & 14 & 71.4 \\
\hline 2011 & 39 & 41.0 & 7 & 14.3 & 7 & 42.9 & 25 & 20.0 & 14 & 78.6 \\
\hline 2012 & 33 & 39.4 & 1 & 100.0 & 6 & 50.0 & 18 & 22.2 & 15 & 60.0 \\
\hline 2013 & 49 & 40.8 & 4 & 50.0 & 19 & 42.1 & 36 & 30.6 & 13 & 69.2 \\
\hline 2014 & 47 & 36.2 & 10 & 20.0 & 16 & 25.0 & 26 & 15.4 & 21 & 61.9 \\
\hline Total & 355 & 36.6 & 69 & 30.4 & 82 & 34.6 & 236 & 23.3 & 119 & 63.0 \\
\hline
\end{tabular}

*Procedures performed in Bernhoven hospital by the same surgeon from 2006-2008

The results of the logistic regression analysis showed that the only significant variable related to a higher PSM rate was pT3 tumor stage (OR 5.35, 95\% CI 3.18-8.98). Increasing PSA, a high risk according to d'Amico and nerve sparing procedures (both unilateral and bilateral) were not significantly related to postoperative PSM (table 3).

\section{PSM Rates in Literature}

We found eight publications from Belgian or Dutch centers on PSM after radical prostatectomy on PubMed. We compared the type of surgery and the numbers performed. Data from all surgical techniques are available: open retropubic RP, laparoscopic RP, endoscopic extraperitoneal RP and robot assisted laparoscopic RP. Numbers treated by RP vary from 51-497 per center. The total PSM-rate is available for all studies and varies from 16-53.2 $\%$. The study of Van Poppel, et al. [13] was excluded from this variation looking at the total PSM-rate because this study only included pT3-tumors. Data for the pT2-stage show a variation in PSM from 2.5-40.3\% and for the pT3-stage from 34.5-77.8 \%. (Table 4). 
Table 3: Logistic regression of PSM in the MMC series.

\begin{tabular}{|l|l|l|l|}
\hline Risk factor & OR & 95\% CI \\
\hline PSA & 1.017 & $0.99-1.04$ & 0.160 \\
\hline High risk d'Amico & 1.652 & $0.91-3.02$ \\
\hline Nerve sparing procedure & 1.722 & $0.94-3.15$ & 0.102 \\
\hline pT3 tumor stage & 5.345 & $3.18-8.98$ & 0.077 \\
\hline
\end{tabular}

Table 4: Summary of publications of PSM in radical prostatectomy in the Benelux.

\begin{tabular}{|c|c|c|c|c|c|c|}
\hline \multirow{2}{*}{ References } & \multirow{2}{*}{ Origin } & \multirow{2}{*}{ Type } & \multirow{2}{*}{$\mathbf{N}$} & \multicolumn{3}{|c|}{ PSM rate \% } \\
\hline & & & & Total & pT2 & pT3 \\
\hline Mottrie, et al. [9] & Belgium & RALP & 184 & 16 & 2.5 & 38 \\
\hline Klaver et al. [10] & Netherlands & RALP & 400 & 19.2 & 13.4 & 34.5 \\
\hline Santeon 1. [11] & Netherlands & LRP & 94 & 53.2 & 40.3 & 72.2 \\
\hline Santeon 2 [11] & Netherlands & RALP & 497 & 40.6 & 30.3 & 57.8 \\
\hline Santeon 3 [11] & Netherlands & LRP & 378 & 35.2 & 26.7 & 69.9 \\
\hline Roumeguere, et al. [12] & Belgium & RRP & 77 & 40 & $7.3^{*}$ & 77.8 \\
\hline Roumeguere, et al. [12] & Belgium & LRP & 230 & 26 & $7.8^{*}$ & 52.9 \\
\hline Joniau, Van Poppel, et al. [13] & Multi - Belgium & RRP & 51 & 62.7 & 0 & 62.7 \\
\hline Wijerman, et al. [14] & Netherlands & LRP/EERPE & 243 & 49.4 & - & - \\
\hline \multirow{3}{*}{$\begin{array}{l}\mathrm{NVU}^{*}[15] \\
\text { "incomplete database with selection bias }\end{array}$} & Netherlands & LRP & 629 & 40.2 & 27.3 & 61.3 \\
\hline & & RRP & 237 & 25.3 & 13.5 & 52.2 \\
\hline & & RALP & 3276 & 30.3 & 21.7 & 52.6 \\
\hline $\begin{array}{l}\text { Fossion, et al. [22] } \\
\text { "current series }\end{array}$ & Netherlands & EERPE & 355 & 36.6 & 23.3 & 63.0 \\
\hline
\end{tabular}

We included data of the national database of the Dutch Association of Urology (NVU) [15] which have been published in 2014. These data show a large variation in PSM between the different surgical approaches. Total PSM-rate was $40.2 \%$ for the laparoscopic approach, $30.3 \%$ for the robot assisted approach and $25.3 \%$ for open surgery. These data have been gathered since 2006 , first on voluntary base and since 2013 the registration is obligatory. In 2014, 4142 RP procedures have been collected in total and were analyzed over a period of eight years.

\section{Discussion}

Our findings concerning the influence of higher tumor stage on the incidence of PSM are consistent with results in literature. The results show that outcome is surgeon specific and tumor stage is a proven risk factor for the incidence of PSM. This is not surprising due to the anatomic location of the prostate; it has expanded surrounding structures (rectum, bladder, sphincter, and symphysis) $[2,4]$. Radical removal of an invasive tumor in the bladder neck or surrounding muscles (pT3) is more complicated than a tumor which is restricted to the prostate only (pT2) [3]. Though nerve sparing procedures could hypothetically complicate malignancy free margins, we did not find any relation with increasing PSM in this group. Our PSM rates are comparable with other centers in literature and with other centers in the Benelux [9-15].

\section{Publications on PSM}

When analyzing the rates of other centers, we should remark that the PSM rate in Belgium is extremely low and comparable with the best centers in the world (Urology Department in Hamburg [18], Memorial Sloan-Kettering Cancer Center) [19]. Especially the PSM rate of pT3 tumors were very low looking at our own rate. It would be interesting to do more research on this remarkable difference between Belgium and the Netherlands and to find out what is the key to this success. Although, a limitation of two Belgian publications is the patient selection of PSM transparency for only pT2 or pT3 tumors, the inclusion of pT1 tumors and the relatively small study population. This makes these studies not ideal for comparison $[12,13]$.

Secondly, we should realize several shortcomings from the Dutch national database [15]. First of all this database has been started in 2006, but is only mandatory from 2013 on. It is clear that several centers have omitted/forgotten to include their data in the database before 2013.We know that annually about 12.000 men are diagnosed with prostate cancer in the Netherlands. From the IKNL-data (Deelrapportage voor prostaatkanker- Januari $2014^{\odot}$ IKNL [20]) we know that about $20 \%$ of these patients underwent a RP; this means annually about 2400 patients. In 2014, 4142 RP procedures have been collected and analyzed over 
a period of eight years. This means that the data, published in 2014, do not represent the quality of RP in the Netherlands, but are only a reflection of the poor participation in quality analysis (4.142 out of +/- 19.200 RP performed over 8 years) when physicians are not obliged to participate in a national database.

Another remarkable finding is the proportion of RALP-data. These are overwhelming in comparison to the other surgical techniques (open RP and EERPE). However, from literature we understand that PSM outcome is similar, regardless the approach which was used [21]. One should admit that at least the robotic surgeons are more willing to show their data than others [9$11,15]$.

And a last important remark regarding the interpretation of these data concerns the lack of information on the inclusion criteria for the different techniques. One can imagine the influence of an investment in e.g. a robotic system on the choice for operative approach in comparison to other treatment options as active surveillance, HiFU, brachytherapy and external radiotherapy with curative intention. This phenomenon has two consequences: one is the accelerating effect on the learning curve and thus the surgeon's experience and the other is the positive effect of the lower PSM-rate in small lower stage tumors on the number of PSM.

\section{Interpretation of urologic data}

Data analysis helps to understand and compare outcome with other surgeons and centers. Since 2013 data on RP are gathered by the national urology associations in both the Netherlands and Belgium. Still those data are not available for both patients and insurance companies. One can understand that the latter groups need adequate help for careful and correct interpretation of these data. This can become the task for the experts of the national urology association itself. An annual quality report on $\mathrm{RP}$, together with an explanation for those data (inclusion criteria and effect of these criteria on the PSM rate), which are hard to interpret for laymen, would help to improve patients quality of care. In the meanwhile, insurance companies have already accessed these data, judging medical centers and surgeons on their performance. These companies decide whether these urology departments are competent enough to perform RP surgery, although they interpret these numbers without any medical background and without the knowledge of the inclusion criteria. One can conclude that the content of national databases and their publications on outcome is very fragile information.

Still there is one major bias in gathering these data: it is the person or organization which collects the data. And this is also the shortcoming of our study. Impartiality and capability should always be insured, and this is nowadays not the case. One should realize that all reports (e.g. pathology, operation, complications...) should be standardized and according the same grading systems (e.g. ASA-classification, TNM-classification, Clavien - Dindo classification...) to make a comparison fair and possible. A crucial role can be played by the national cancer registry (IKNL in the Netherlands and National Kanker Register in Belgium) to collect these data in a uniform template and insure impartiality.
A single surgeon study is a good way to analyze a learning curve and individual progression. It shows that achieving radical prostatectomy with no PSM, is an accomplishment that can be taught. However, the limitation of this study design is that it contains results of one urologist only and is therefore less applicable to others. We tried to compensate for this fact by including other publications on PSM of other urologists or centers. Another possible limitation is confounding, because we only analyzed urologic variables (such as d'Amico risk and pathological tumor stage) regarding PSM, and did not take ASAscore or perioperative complications into account. These factors might have influenced the ability of extensive procedure and therefore PSM.

Several patient characteristics can influence peri- and postoperative outcome, both for oncologic and functional results $[16,22]$. Transparency of these data is essential for correct interpretation of the outcome data. Still a standardized format is not available. Research need to be done to quantify and assign those parameters which do influence patient outcome after surgery. The criteria have evolved from the trifecta, to the pentafecta and now octofecta to demonstrate quality of care in RP. Besides, not in all patients with a PSM disease recurrence automatically occurs. According to a study conducted by van Oort, et al. [23] and Pettenati, et al. [5], the five-year risk of developing biochemical recurrence was approximately onethird in the PSM patients. Note that biochemical recurrence is not the same as disease recurrence and hence could still not be of any clinical relevance to the patient. When disease recurrence does occur, treatment options are still available, like salvage radiotherapy. This multimodality therapy has proved to offer still a good chance of complete remission during the EORTC trial [24] and the SWOG 8794 study [25].

\section{Conclusion}

Our data confirm the relation between the tumor stage and the incidence of positive surgical margins after radical prostatectomy in our department. It was also proved that a learning curve contributes to lower PSM rates.

Comparison with other centers is difficult because of the limited existing publications on this subject in the Benelux. Uniform, mandatory registration in a national database of all performed radical prostatectomy's can be the key for annual reporting on quality of surgical care, thus improving transparency towards patients. The national urology association should help both patients and insurance companies in the interpretation of the published report.

\section{Acknowledgements}

We would like to thank Dr. K. de Laet, urologist in the Máxima Medical Centre, for his useful comments when we asked him to review our article. Also we would like to show our gratitude to the urology team of the Máxima Medical Centre for their support of our clinical research.

\section{Declarations}

The authors certify that there is no conflict of interest with 
any financial, research, and academic organization, with regards to the research work discussed in the manuscript.

Since this work contains data gathered retrospectively using an anonymous database, no effort regarding ethical approval was conducted.

\section{References}

1. Nicolas Mottet, P J Bastian, J Bellmunt, Michel Bollad, Steven Joniaue, T.H. van der Kwast, et al. Guidelines on prostate cancer. European Urology. 2014;65(1):124-137.

2. Mottrie A, Gallina A, De Wil P, David Thüer, Giacomo Novara and Vincenzo Ficarra. Balancing continence function and oncological outcomes during robot-assisted radical prostatectomy (RARP). BJU International. 2011;108(6):999-1006. DOI: 10.1111/j.1464410X.2011.10529.x

3. Oncoline. National guidelines for cancer care in prostate cancer. Dutch Association Urology (last update 04-16-2014). available from: http:// www.oncoline.nl/prostaatcarcinoom

4. D. Van Den Ouden, W.C.J. Hop, R. Kranse, F.H. Schröder. Tumorcontrole bij patiënten met klinisch niet-gemetastaseerd prostaatcarcinoom, behandeld met radicale prostatectomie: een prospectief onderzoek. Ned Tijdschr Geneeskd. 1998;142(7):349-354.

5. Caroline Pettenati, Yann Neuzillet, Camelia Radulescu, Jean-Marie Hervé1, Vincent Molinié, Thierry Lebret. Positive surgical margins after radical prostatectomy: What should we care about? World J Urol. 2015;33(12):1973-1978. DOI: 10.1007/s00345-015-1580-x

6. Joshua J. Meeks, James A. Eastham. Radical prostatectomy: Positive surgical margins matter. Urologic Oncology: Seminars and Original Investigations. 2013;31(7):974-979. DOI:dx.doi.org/10.1016/j. urolonc.2011.12.011

7. Wright JL, Dalkin BL, True LD, William J. Ellisa, Janet L. Stanfordc, Paul H. Lange et al. Positive surgical margins at radical prostatectomy predict prostate cancer specific mortality. J Urol. 2010;183(6):22132218. doi:10.1016/j.juro.2010.02.017

8. Pierre I. Karakiewicz, James A. Eastham, Markus Graefena, Ilias Cagiannosa, Phillip D. Strickerd, Eric Kleine, et al. Prognostic impact of positive surgical margins in surgically treated prostate cancer: Multiinstitutional assessment of 5831 patients. Urology. 2005;66(6):12451250. doi:10.1016/j.urology.2005.06.108

9. Alexander Mottrie, Peter Van Migem, Geert De Naeyer, Peter Schatteman, Paul Carpentier, Etienne Fonteyne. Robot-Assisted Laparoscopic Radical Prostatectomy: Oncologic and Functional Results of 184 Cases. European urology. 2007;52:746-751. doi:10.1016/j. eururo.2007.02.029

10. Rambaran SS, Van Den Ouden D, Kliffen M, De Lange DCD, Klaver OS. Robot-assisted laparoscopic radical prostatectomy in a general hospital : perioperative parameters and short-term functional and oncologic results from the first 400 patients. Journal of Urology. 2011;1(6):126-132. DOI:10.1007/s13629-011-0058-x

11. Santeon. Care outcomes. Outcome indicators for prostate cancer and lung cancer. Results Catharina Hospital, St. Antonius Hospital and Canisius-Wilhelmina Hospital . 2013. Available from: http://www. zorgvooruitkomst.nl/uitkomstenboek2013.pdf

12. Roumeguere T, Bollens R, Vanden Bossche M, Dan Rochet, David
Bialek, Paul Hoffman, et al. Radical prostatectomy: a prospective comparison of oncological and functional results between open and laparoscopic approaches. World J Urol. 2003;20(6):360-366. DOI: 10.1007/s00345-002-0306-z

13. Joniau S, Hsu CY, Gontero P, Spahn M, Van Poppel H. Radical prostatectomy in very high-risk localized prostate cancer: Longterm outcomes and outcome predictors. Scand J Urol Nephrol. 2012;46(3):164-71. DOI:10.3109/00365599.2011.637956

14. Coenen J, Roovers A, Koot R, Wijerman P. Pathologische kenmerken van het laparoscopische radicale prostatectomiepreparaat: het belang van snijvlakspecificaties beschreven door de patholoog. Tijdschrift voor Urologie. 2012;2(1):11-16. DOI: 10.1007/s13629-012-0004-6

15. Van Der Poel H, Wijsman B. Prostatectomy quality registration in recent years. Association News. Journal of Urology. 2014;5-7.

16. Jacobs RAL, Fossion LMCL. A critical analysis of the first 218 endoscopic extrapertioneal radical prostatectomy procedures in a Dutch nonacademic hospital. Tijdschrift voor Urologie. 2012;2(8):197-204. DOI: 10.1007/s13629-012-0105-2

17. Stolzenburg JU, Do M, Pfeiffer H, König F, Aedtner B, Dorschner W. The endoscopic extraperitoneal radical prostatectomy (EERPE): technique and initial experience. World J Urol. 2002;20(1):48-55. DOI: 10.1007/s00345-002-0265-4

18. Ofer Yossepowitch, Alberto Briganti, James A. Eastham, Epstein J, Graefen M, et al. Positive Surgical Margins after Radical Prostatectomy: A Systematic Review and Contemporary Update. European Urology. 2014;65(2):303-313. doi: 10.1016/j.eururo.2013.07.039

19. Andrew J. Vickers, Fernando J. Bianco, Angel M. Serio, Eastham JA, Schrag D, Klein EA, et al. The Surgical Learning Curve for Prostate Cancer Control after Radical Prostatectomy. J Natl Cancer Inst. 2007;99(15):1171-1177.

20. Integraal Kankercentrum Nederland. Deelrapportage voor prostaatkanker. 2014 IKNL.

21. Ficarra V, Novara G, Artibani W, Cestari A, Galfano A, Graefen M, et al. Retropubic, Laparoscopic and Robot-Assisted Radical Prostatectomy: A Systematic Review and Cumulative Analysis of Comparative Studies. Eur Urol. 2009;55(5):1037-1063. doi: 10.1016/j.eururo.2009.01.036.

22. K De Laet, Fossion EMCL, Nanlohy-Manuhutu EL, Levens WJ, Karaoglu L. Jaarverslag Maatschap Urologie MMC (2013):33-50. Available from: http://ic.mmc.nl/index.php/media/urologie/uro_documenten/ jaarverslag_2013

23. Van Oort IM, Bruins HM, Kiemeney LA, Knipscheer BC, Witjes JA, Hulsbergen-Van De Kaa CA. The length of positive surgical margins correlates with biochemical recurrence after radical prostatectomy. Histopathology. 2010;56(4):464-471. DOI: 10.1111/j.13652559.2010.03497.x

24. Bolla M, Van Poppel H, Collette L, van Cangh P, Vekemans K, Da Pozzo $\mathrm{L}$, et al. Postoperative radiotherapy after radical prostatectomy: a randomised controlled trial (EORTC trial 22911).Lancet. 2005;366(9485):572-578.

25. Ian M Thompson, Catherine, Tangen M, Jorge Paradelo, Lucia MS, Miller G, et al. Adjuvant Radiotherapy for Pathological T3N0M0 Prostate Cancer Significantly Reduces Risk of Metastases and Improves Survival: Long-Term Followup of a Randomized Clinical Trial. J urology. 2009;181(3):956-962. doi: 10.1016/j.juro.2008.11.032. 